\title{
Institutionelle Regelungen im Entwicklungsprozess Einführung zum Themenheft
}

\section{Ulrike Müller-Böker, Zürich}

Im Mai 2000 fand in Zürich die Tagung des Geographischen Arbeitskreises Entwicklungstheorien statt, seit seiner Gründung im Jahre 1976 das erste Treffen in der Schweiz! Die Beiträge des Geographica Helvetica Themenhefts stammen aus der Feder von Teilnehmern und Teilnehmerinnen dieser Tagung und orientieren sich an folgenden thematischen Schwerpunkten:

- Die Neue Institutionenökonomie und Dezentralisierung als theoretisches und politisches Konzept (W. ThOMI, T. RAUCH)

- Institutionelle Regelungen im Konflikt um Land (M. Coy, M. Neuburger)

- Institutionelle Regelungen im Konfliktfeld «Naturschutz und Entwicklung» (M. RöPER, N. BACKHAUS und M. Kollmair).

Institutionen - im Sinne von D. NoRTH (1990:3) begriffen als «...the rules of the game in a society, or, more formally ...the humanly devised constraints that shape human interaction» - bilden den gemeinsamen Ausgangspunkt der Beiträge.

\section{Warum das Thema Institutionen?}

Das Thema Institutionen ist nicht neu. Viele geographische Arbeiten, nicht nur im Kontext der Entwicklungsforschung, haben sich implizit und explizit mit Institutionen aller Art befasst. Ein breites Spektrum von theoretischen Zugängen aus den Nachbarwissenschaften, insbesondere der Ökonomie liegt vor (vgl. Texte zur Vorbereitung der Tagung, GAE 2000). An dieser Stelle sei auf drei wichtige Namen verwiesen: D. North, A. Sen und E. Ostrom, deren Arbeiten nicht nur mit Debatten über «Entitlement» und «Institutionen» verknüpft sind, sondern auch mit Zürich. Sie sind alle Ehrendoktoren der Universität!

In der Regel rechnet man mit etwa zehn Jahren Verzögerung, bis neue wissenschaftliche Fragestellungen und Ergebnisse in anderen «Milieus» ankommen. Vieles deutet darauf hin, dass die Institutionen - im Gefolge von Partizipation, «Good Governance» und Strukturanpassungsprogrammen - endgültig die Entwicklungspolitik erreicht haben und nun aktuell sind.
In den einschlägigen Publikationen der Entwicklungszusammenarbeit mehrt sich entsprechend die Zahl der Beiträge, die sich mit Institutionen befassen und auf die grosse Bedeutung der Institutionenförderung verweisen.

Es lässt sich also eine strategisch bedeutsame Verschiebung in der Entwicklungspolitik diagnostizieren. «Institutionenförderung statt Projektförderung» (KochendöRfER-LucIUS \& VAN DER SAND 2000), d.h. nicht mehr «asset creation» (Bildung von Vermögen oder Fähigkeiten) steht im Vordergrund, sondern die Schaffung von Strukturen, die Zielgruppen Zugang zu Ressourcen ermöglichen oder Wachstum fördern. Die Hilfe zur Veränderung entwicklungsbehindernder Institutionen bzw. die Förderung von institutionellen Kapazitäten (Entscheidungsmechanismen und Organisationsformen) stehen auf der aktuellen entwicklungspolitischen Agenda.

Verfolgt man diesen Diskurs um die Institutionen kritisch, stellt sich die Frage, aus welchen Erfahrungen, theoretischen Konzeptionen und versteckten Positionen, aus welchen normativen Vorgaben dieser Nahrung erhält. Denn im entwicklungspolitischen Kontext werden Institutionen nicht wertfrei analysiert, sondern auf ihre Funktionstüchtigkeit, auf ihre Fähigkeit, bestimmte Ziele und Aufgaben zu verfolgen, betrachtet, bewertet oder gefördert. Die Neue Institutionenökonomie und die «main stream»-Entwicklungspolitik werten dabei «gut funktionierende» Institutionen als Motor und Voraussetzung für wirtschaftliches Wachstum und Demokratisierungsprozesse. Die Weltbank widmet entsprechend den nächsten Weltentwicklungsbericht (2001/02) dem Thema: «Institutionen und wirtschaftliche Entwicklung».

Wenn man «Entwicklung» jedoch als einen - häufig konfliktären - Prozess begreift, an dessen Gestaltung und Ausrichtung verschiedene Gruppen strategisch beteiligt sind, dann bedürfen formelle und informelle institutionelle Regelungen zunächst der wissenschaftlichen Analyse. Deren Ergebnisse können dann wegleitend für politisches Handeln sein. Normativ gesehen wäre als Ziel zu formulieren, gesellschaftliche Disparitäten und Konflikte zu lösen, indem institutionelle Regelungen gefunden und gefördert werden, die einen gerechten und nachhaltigen Zugang zu Ressourcen fördern. Nicht mehr der Mangel an Ressourcen oder technisch-organisatorischen Möglichkeiten (Activa) steht dabei im Vordergrund, sondern die 
institutionellen Regelungen, die für mangelhafte und ungerechte Zugangswege sorgen.

Die geographische Entwicklungsforschung befasst sich in diesem Kontext mit klassischen Forschungsfragen wie z.B. Dezentralisierung, Konflikte um den Zugang zu Land oder Konflikte im Naturschutz. Formelle und informelle, lokal bis global verankerte Institutionen werden analysiert. Das Nebeneinander verschiedener Regelungen - ein Indikator für geringen gesellschaftlichen Konsens, rechtliche Unsicherheit, mangelnde Transparenz und für virulente gesellschaftliche Konflikte - steht im Vordergrund. Wenn also der Fokus auf Institutionen gesetzt wird, dann mit dem Ziel, Wege und Strategien zu einer Differenzierung, Konkretisierung und einem besseren Verständnis (und vielleicht sogar Lösung) der gesellschaftlichen Probleme zu entwickeln.

\section{Die Beiträge}

Die «Neue Institutionenökonomie» (NIÖ) und dabei in erster Linie die Arbeiten von D. NoRTH werden in den Beiträgen von W. Thом und T. Rauch aus verschiedenen Perspektiven aufgegriffen. Einen guten Einstieg in die Grundzüge der NIÖ bietet zunächst der Beitrag von W. ThомI, der im weiteren auch einen neuen, auf der NIÖ basierenden Interpretationshintergrund für Dezentralisierungsprozesse entwickelt. Demgegenüber bemängelt $T$. RAuCH an der NIÖ, dass die institutionelle Sphäre als eine von gesellschaftlichen Entwicklungsprozessen weitgehend unabhängige, ökonomische Variable losgelöst betrachtet wird. Gleichzeitig bietet dieser Beitrag eindrückliche Einsichten in die aktuelle politische Entwicklung Südafrikas und zeigt exemplarisch, dass es kein allgemeingültiges politisch-administratives Modell der Dezentralisierung gibt.

Zwei Beiträge befassen sich mit den institutionellen Regelungen im Konflikt um Land. M. CoY gibt einen Überblick zum Stand der Diskussion in den Entwicklungsländern und greift zentrale Aspekte formeller und informeller Institutionen auf, die die Landfrage mitbestimmen. M. NeUBURGER untersucht die unterschiedlichen Bodenrechtstypen, die in Brasilien aufeinandertreffen, und analysiert in drei Fallbeispielen aus der Pantanalregion deren Auswirkungen hinsichtlich Verwundbarkeit und kleinbäuerlichen Überlebensstrategien.

Institutionelle Regelungen im Konfliktfeld «Naturschutz und Entwicklung» stehen im Zentrum der Beiträge von M. RöPER und N. Backhaus und M. Kollmair. M. RÖPER entwickelt - indem sie verschiedene sozialgeographische Ansätze kombiniert - ein Modell zur
Entstehung und zum Verlauf von Konflikten bei der Planung und Einrichtung von Naturschutzgebieten in Entwicklungsländern. Naturschutzgebiete werden dabei als eine Form der Institutionalisierung des Zugangs zu und der Kontrolle von Raum begriffen. Welche unterschiedlichen Verläufe dieser Institutionalisierungsprozess nehmen kann, wird am Beispiel von drei Schutzgebieten in West-Brasilien exemplifiziert. Im Beitrag von N. Backhaus und M. Kollmair geht es vor allem um die Fragen, welche Ansprüche an Nationalparks gestellt werden, bzw. welche Verfügungsrechte an den Ressourcen bestehen und welche Institutionen dabei eine Rolle spielen. Der «Environmental Entitlements»Ansatz (Leach, Mearns \& Scoones, 1997) wird als Analyseinstrument bei der Untersuchung von Nationalparks in Malaysia und Nepal eingesetzt.

Das Spektrum der Beiträge des Themenhefts bietet einen Überblick über theoretische und methodische Zugangswege und empirisch untersuchte Problemfelder im Bereich institutioneller Regelungen. Innovativ werden theoretische Erkenntnisse vor allem aus NIÖ und Strukturationstheorie aufgegriffen und in der empirischen Forschung als Analyseinstrumente genutzt und überprüft. Die Bedeutung von institutionellen Regelungen im Entwicklungsprozess gilt als unbestritten, allerdings wird in allen Beiträgen deutlich, dass diese nicht losgelöst von anderen Kontexten und Zielvorgaben betrachtet werden sollten.

\section{Literatur}

GAE (Geographischer Arbeitskreis Entwicklungstheorien) (2000): Reader mit Texten zur Tagung «Institutionelle Regelungen im Entwicklungsprozess» des Geographischen Arbeitskreises Entwicklungstheorien, Zürich, 26.-28. Mai 2000. Konzeption Reader: BEIER, C. \& H. DüRR, Bochum, Zürich.

Kochendörfer-LuCIUS, G. \& K. VAN DER SAND (2000): Entwicklungshilfe vom Kopf auf die Füsse stellen. Institutionenförderung statt Projektförderung. - In: E + Z Entwicklung und Zusammenarbeit 41(4): 96-99.

Leach M., Mearns R. \& I. Scoones (1997): Environmental entitlements: A framework for understanding the institutional dynamics of environmental change. - = IDS Discussion Paper 359, March 1997, Institute of Development Studies at the University of Sussex, Brighton.

NoRTH, D. C. (1990): Institutions, Institutional Change and Economic Performance. - Cambridge, New York, Melbourne: Cambridge University Press.

Prof. Dr. Ulrike Müller-Böker, Geographisches Institut der Universität Zürich, Abteilung Anthropogeographie, Winterthurerstrasse 190, CH-8057 Zürich.

e-mail:boeker@geo.unizh.ch 